\title{
Minimally invasive treatment of iatrogenic complete left ureter obstruction after hysterectomy
}

\author{
Yigit Akin ${ }^{1 *}$, Isil Basara², Aliseydi Bozkurt² \\ ${ }^{1}$ Department of Urology, School of Medicine, Erzincan University, 24040, Erzincan, Turkey. ${ }^{2}$ Department of Radiology, Harput \\ State Hospital, 23050, Elazig, Turkey.
}

\begin{abstract}
The iatrogenic ureter injuries are rare complications and may have serious consequences. The treatment options depend on situations. Pelvic surgeons, keep in mind that this kinds of complications, is very important for diagnosis and treatment, during the surgery. This report presents a case of a patient with iatrogenic left ureteral injury during hysterectomy. The patient visited emergency department of our hospital with the chief complaints of left lomber pain on the 17th day of hysterectomy. After evaluation in emergency clinic, the patient had an endoscopic treatment for iatrogenic ureter injury. The patient is still in follow-up period. We also review the literature and discuss diagnose, treatment, prognosis of iatrogenic ureter injuries. The treatment options are still developing by technology.

(c) 2012 All rights reserved
\end{abstract}

Keywords: hysterectomy, minimally invasive, surgical procedures, ureteral obstruction, wounds, injuries.

\section{Introduction}

The iatrogenic ureteral injuries are rare; this complication is the one of the most important complication in gynecological surgery (1). The injuries almost locate in the distal part of ureter (2). Quick diagnosis and treatment decrease complication rates (3). Also early diagnosis provides the best results for treatment (3). There are a lot of treatment options. When this rare complication applies our clinics, we may prefer minimally invasive treatment options (4-6). Herein, we present a 62-year-old woman with a complete left ureter obstruction which was formed by a suture in her ureter with a previous hysterectomy.

\section{Case report}

On April 2011, a 62-year-old woman with complaints of abdominal pain was admitted to our hospital. She was evaluated in gynecology outpatient

\footnotetext{
* Corresponding author: Yigit Akin, Department of Urology, School of Medicine, Erzincan University, Erzincan/Turkey Tel: +90-506-5334999

e-mail: yigitakin@yahoo.com
}

Submitted: 15 May 2012/ Accepted: 4 August 2012 clinic. She had big submucous myoma uteri and after diagnosis, she had a hysterectomy operation for myoma uteri. On the 17th day of surgery, she had visited emergency department of our hospital with the chief complaints of left lumbar pain and dysuria. Then, physical, laboratory and radiological evaluations were performed. In physical examination she had left lumbar tenderness and pain.

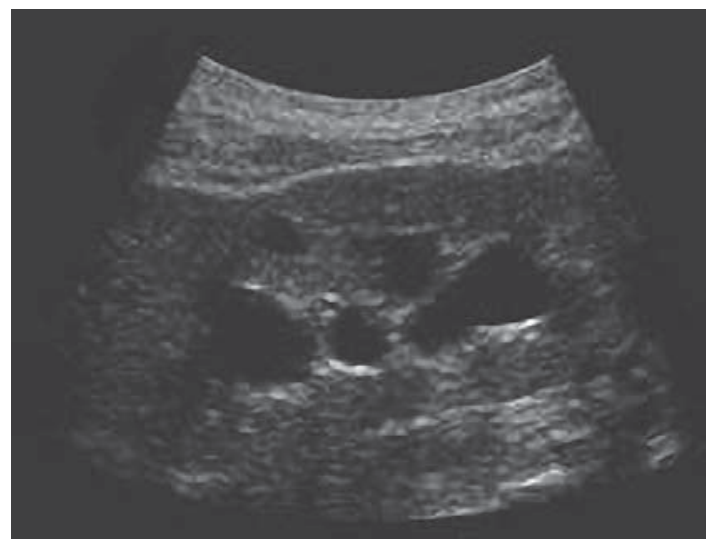

FGURE 1 In gray scale US examination, there are dilatations in all calyx system. The level of dilatation is grade 2-3 hydronephrosis. 


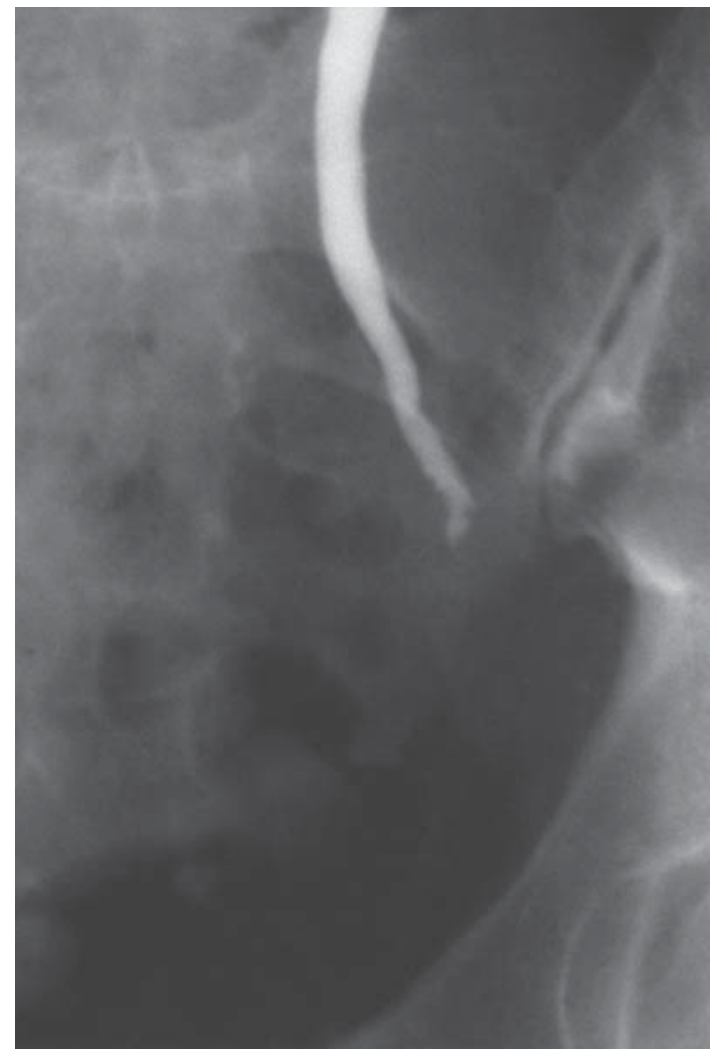

PGURE 2 In IVU, there is complete obstruction in the lower part of left ureter. Additionally, there is mild dilatation in the proximal part of ureter.

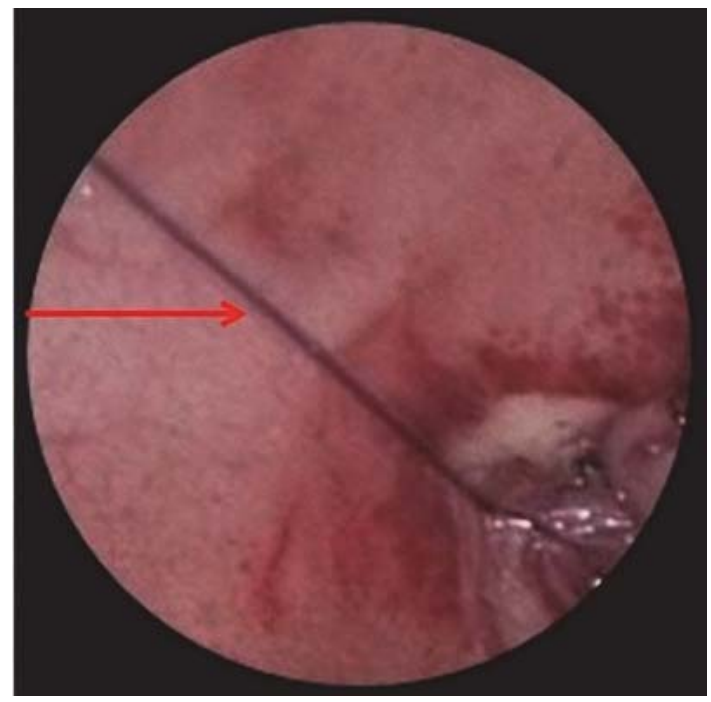

FGURE 3. Endoscopic view of the suture (arrow).

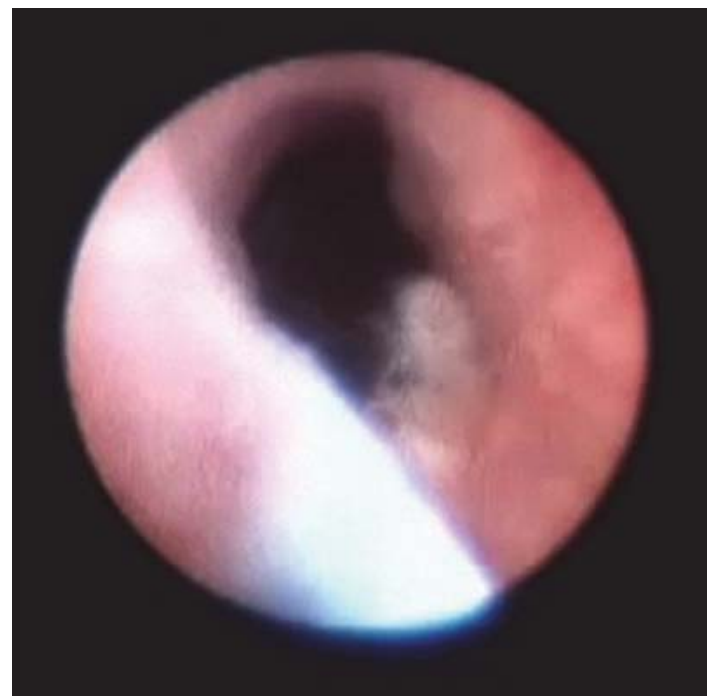

FGURE 4. Ureteroscopic view of ureter after ureterotomy was performed. Guide wire is also in the ureter.

The urine test and serum creatinine were normal. In ultrasonography (US) examination, there was grade 2-3 hydronephrosis with mild proximal left ureter dilatation (Figure 1). After ultrasound (US) examination, intravenous urography (IVU) evaluation was performed in order to find out the exact obstruction localization and the reason of the obstruction (Figure 2). There was not a stone image in the kidney ureter bladder $\mathrm{x}$-ray examination. There was a complete obstruction in the distal part of left ureter, in nephrogram phase of IVU. In the light of thesefindings, we concluded that there was distal left ureter obstruction which was formed iatrogenic after hysterectomy operation and we performed endoscopic evaluation by ureteroscopy. In ureteroscopy, we could reach the suture material at the left distal ureter (Figure 3). After that, we cut the suture material with cold ureterotomy and we reached left kidney with semirigid ureteroscopy by using guide wire (Figure 4). Then double $\mathrm{j}$ stent was put into ureter. She discharged at the 1 st day after the surgery. We took off the double $\mathrm{j}$ catheter after 1st month of the surgery.

\section{Discussion}

Ureteral injury is one of the rare and serious complications of gynecologic surgery. The frequency of ureteral injury following gynecologic 
surgery is approximately $1 \%$, with a higher percentage of injuries occurring during abdominal hysterectomies and partial vaginectomy (1). Nevertheless, when a ureteral injury occurs, quick recognition of the problem and a working knowledge of its location and treatment are essential in providing patients with optimal care. Ureter lies on anterior of psoas muscle and crosses over the iliacs and also crosses anteriorly by the gonadal vessels. Most of the iatrogenic injured cases do not have any identifiable risk factors. Endometriosis, uterus size larger than 12 weeks gestation, ovarian cysts $4 \mathrm{~cm}$ or larger, pelvic radiation therapy, anatomic anomalies of urinary tract, ovarian masses, inflammation of pelvis, pelvic malignancy are the some of the risk factors which may disrupt of normal pelvic anatomy (2). The reasons of iatrogenic ureter injury are, misapplication of a clamp, ligation with suture, transection of ureter, ureteral ischemia form electrocoagulation, resection of segmental ureter, secondary obstruction of ureter by angulation. Also the combination of all of these reasons may lead the iatrogenic ureter injury (5). Ureteral injury may be divided as recognized or unrecognized during surgery. When the iatrogenic injury was diagnosed during the surgery, the time of urologist starts-up in operation. The injury may be treated best by urologist with ureteral repair during the same operation. Unilateral ureteral injuries are occurred nearly $75 \%$ of the cases after the operation (7). If the injury of ureter has occurred and has not been recognized, it may cause flank pain, chronic ureteral obstruction or formation of fistulas. In our case, the iatrogenic injury had occurred after the surgery. Then, the injury was diagnosed quickly and it was treated endoscopic minimally invasive surgery. As a result of developing technology, laparoscopic and robotic-assisted surgery have been included to the surgery modalities. Same as open pelvic surgery, in laparoscopic and robotic-assisted surgery, surgeons should keep in their minds the iatrogenic ureter injury (8). If the ureteral injury can be diagnosed intraoperative, intravenous administration of indigo carmine or methylene blue with furosemide may help to localize a ureteral injury (1). Extravasation of blue dye indicates ureteral discontinuity. Also fluoroscopy may help us by administration of contrast substance in ureter and show us the injury or obstruction zone in ureter. If the ureteral injury has occurred after surgery, laboratory studies including complete blood count, a electrolyte panel with serum creatinine and blood urea nitrogen are needed to distinct for possible infection and renal failure. In radiological evaluation, renal US, if the patient's serum creatinine levels are normal IVU, abdominal and pelvic computed tomography with intravenous contrast may be used. Although renal US is the best non-invasive method to visualize the kidney and shows hydronephrosis it cannot be used to assess kidney function or the continuity of the ureter. Urologists use the IVU to evaluate for continuity of the ureter in cases of ureteral injury. Unlike renal ultrasonography and a retrograde ureteropyelography, IVU is used to assess for function of the ipsilateral kidney and the drainage of the ureter in a series of sagittal images. Hydronephrosis, ureteral integrity, and any extravasation may usually be seen with IVU. CT scan can also be used to assess for both function of the ipsilateral kidney and drainage of the ureter. CT scanning has the advantage of imaging for concomitant conditions at the same time (5). The treatment options depend on situation of the cases. There is no exact medical treatment options for iatrogenic ureter injuries but some conditions such as infection, renal failure which belongs to ureteral injury should be treated medically. The surgical treatments may range from minimally invasive treatments to ureteroneocystostomy. The most common open, laparoscopic or roboticassisted surgical treatments for ureteral injury are simple removal of a ligature, ureteral stenting, ureteral resection and ureteroureterostomy, transureteroureterostomy, and ureteroneocystostomy (9-11). Wolf et al. (12) reported long term excellent results of endoureterotomy. In our case, we simply removed the ligated suture by endouretetomy and put ureteral stent. If the patient's situation is not suitable for treatment of ureteral injury the urinary diversion should be perform by percutaneous nephrostomy catheter. This provides decompression of closed urinary system. If the obstruction or injury is managed by minimally invasive endoscopic surgery and additionally ureteral stent is put in to ureter, the stent may 
be put off 15th day of surgery after uretral healing and regression of renal hydronephrosis. If psoas hitch, boari flep, ureteroneocystostomy or other open surgical procedure are performed, urethral catheter may be taken off 10th day of surgery and ureteral stent may be put off 15 th-30th day of surgery. In 3rd month of the surgery, the upper urinary system imaging should be performed (11). We conclude that, this case shows the importance of early diagnosis and treatment of iatro- genic ureter injury which occurred after surgery. Also this case emphasizes the significance of ureteroscopy which is a minimal invasive and effective method in the diagnosis and treatment. In the future, the use of new minimally invasive technologies will be able to change the management of iatrogenic ureter injuries.

\section{Competing interests}

Authors declare no conflict of interest.

\section{References}

(1) Selzman AA, Spirnak JP. Iatrogenic ureteral injuries: a 20 - year experience in treating 165 injuries. J Urol 1996;155(3): 878-881.

(2) Payne CK. Ureteral injuries in the female: fistulas and obstruction. In: Raz S (ed). Female Urology. 2nd ed. Philadelphia: W.B. Saunders, 1996. pp. 507-20.

(3) Gilmour DT, Dwyer PL, Carey MP. Lower urinary tract injury during gynecologic surgery and its detection by intraoperative cystoscopy. Obstet Gynecol 1999; 94(5): 883889.

(4) Mate-Kole MO, Yeboah ED, Affram RK, Ghosh TS. Anuric acute renal failure due to bilateral accidental ureteric ligation during abdominal hysterectomy. Int J Gynaecol Obstet $1993 ; 41(1): 67-73$.

(5) Ghali AM, El Malik EM, Ibrahim AI, Ismail G, Rashid M. Ureteric injuries: diagnosis, management, and outcome. J Trauma 1999; 46(1):150-158.

(6) Armenakas NA. Current methods of diagnosis and management of ureteral injuries. World J Urol 1999; 17(2):78-83.

(7) Serin SI, Ekmekcioglu O, Yirmibes U, Aygen E. Jinekolojik ve Obstetrik Ameliyatlarda Iyatrojenik Uriner Sistem Yaralanmaları. Turkiye Klinikleri J Gynecol Obst 2001; 11(1):37-41.

(8) Kalisvaart JF, Finley DS, Ornstein DK. Robotic-assisted repair of iat- rogenic ureteral ligation following robotic-assisted hysterectomy. JSLS. 2008;12(4):414-416.

(9) Mathews R, Marshall FF. Versatility of the adult psoas hitch ureteral reimplantation. J Urol. 1997; 158(6): 2078-2082

(10) Konigsberg H, Blunt KJ, Muecke EC. Use of Boari flap in lower ureteral injuries. Urology. 1975;5(6):751755

(11) Koo HP, Bloom DA. Lower ureteral reconstruction. Urol Clin North Am. 1999; 26(1):167-173.

(12) Wolf JS Jr, Elashry OM, Clayman RV. Long-term results of endoureterotomy for benign ureteral and ureteroenteric strictures. J Urol. 1997;158(3):759-764. 